\title{
A STEM/EELS study of interfaces in delafossite-based quantum heterostructures
}

Sangmoon Yoon ${ }^{1}$, Jong Mok Ok ${ }^{1}$, Mina Yoon ${ }^{1}$, Sinchul Yeom ${ }^{2}$, Tomohiro Ichiba ${ }^{1}$, Fernando A. Reboredo ${ }^{1}$, Amanda Huon ${ }^{1}$, Andrew R. Lupini ${ }^{1}$ and Ho Nyung Lee ${ }^{1}$

${ }^{1}$ Oak Ridge National Laboratory, United States, ${ }^{2}$ University of Tennessee, United States

Metallic delafossite oxides $A B \mathrm{O}_{2}(A=\mathrm{Pd}$ or $\mathrm{Pt})$ have attracted considerable attention for their natural heterostructures that highlight the combination of nearly free electrons and band/Mott insulating states. Thus, this materials system is a great platform for exploring exotic quantum phenomena emerging from highly conductive electrons manipulated by the extreme anisotropy or by the Berry phase (fictitious magnetic field), e.g., hydrodynamic electron flow ${ }^{1}$, maximal Rashba-like spin splitting $^{2}$, and unconventional anomalous/topological Hall effects ${ }^{3-4}$. To date, all these physical phenomena have been observed only in bulk single crystals.

Such intriguing properties have motivated recent attempts to grow delafossite thin films or heterostructures using the state-of-the-art molecular beam epitaxy and pulsed laser deposition techniques. The epitaxial growth of metallic delafossites not only provides a route to utilize their diverse properties in future technological applications, but also enables the design of artificially-structured delafossite metals, in which new quantum phenomena may emerge. Despite vast research efforts, the synthesis of highquality delafossite metals in thin-film forms still remains a challenge. This is mainly due to the volatile nature of $\mathrm{PdO}$, which makes the initial nucleation hard as the surface adsorption is limited on structurally dissimilar substrates, such as $\mathrm{SrTiO}_{3}(111)$ and $\mathrm{Al}_{2} \mathrm{O}_{3}$ (0001). Control of the chemistry and structure of the interface to allow the growth of high-quality delafossite thin films will be an important milestone for exploring new quantum phenomena in delafossite-based quantum heterostructures.

In this talk, we will show that the use of a $\mathrm{CuCrO}_{2}$ buffer layer is a great way to control the interface delafossite symmetry and facilitate the epitaxial growth of high-quality $\mathrm{PdCrO}_{2}$ thin films. ${ }^{5}$ Interestingly, the thinner the buffer layer, the better the crystal quality and transport properties. Meanwhile, confusingly, the buffer layers are not visible in HAADF STEM images of $\mathrm{CuCrO}_{2}$-buffered $\mathrm{PdCrO}_{2}$ thin films (Figure 2). To uncover the mechanism of how the $\mathrm{CuCrO}_{2}$ buffer layer manipulates the chemistry and structure of the interface, we systematically examine the microstructural evolution of a $\mathrm{CuCrO}_{2}$ thin film when $\mathrm{PdCrO}_{2}$ is deposited on the top surface via atomic-scale STEM/EELS experiments. We directly observe that $\mathrm{Cu}$ ions are pumped out to the surface while $\mathrm{Pd}$ ions are sucked into the $\mathrm{CuCrO}_{2}$ thin films during the $\mathrm{PdCrO}_{2}$ deposition. We further find that the addition of excess electrons from Pd-terminated surfaces, dominantly exposed during the deposition, provides a strong driving force to induce the reaction. Our STEM/EELS study reveals the $\mathrm{CuCrO}_{2}$ buffer layer plays a sacrificial role as it provides the interface delafossite symmetry to $\mathrm{PdCrO}_{2}$, opening the door to the successful growth of high-quality $\mathrm{PdCrO}_{2}$ epitaxial thin films with excellent transport properties. 
Metallic Delafossite Heterostructure: Promising platform to study exotic quantum behavior
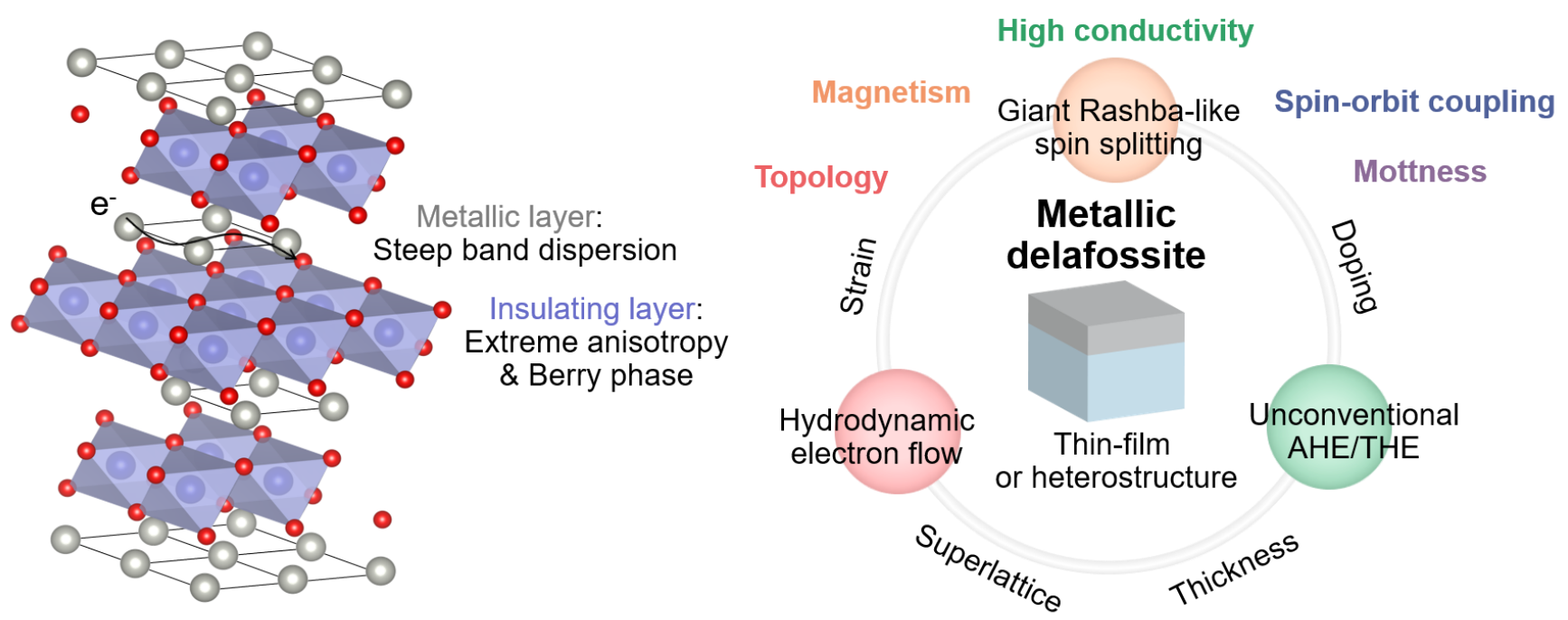

Figure 1. Figure 1. Perspective of metallic delafossite heterostructures as an emerging quantum material. Metallic delafossites are a great platform to explore exotic quantum phenomena arising from nearly free electrons manipulated by the extreme anisotropy or by the Berry phase. The epitaxial growth of metallic delafossites may provide many scientific and technological opportunities.
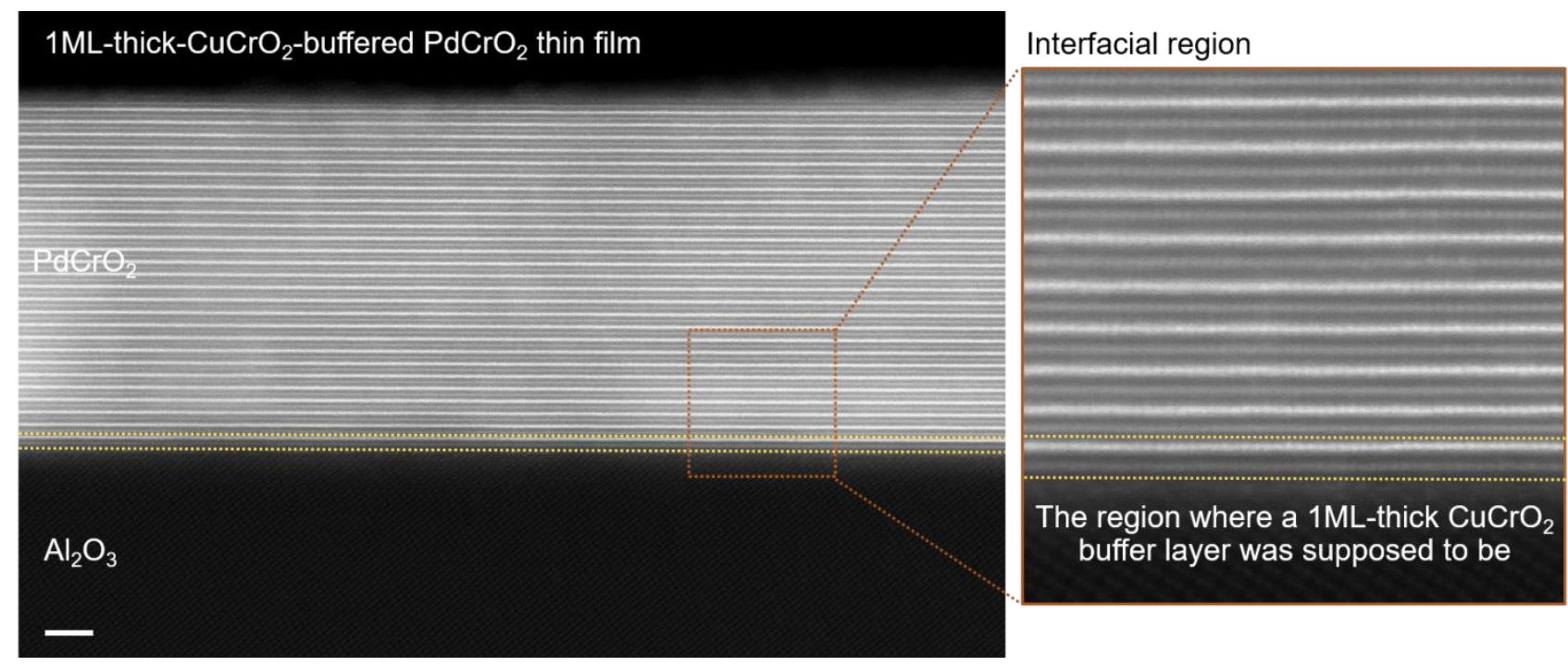

Figure 2. Figure 2. High-resolution HAADF STEM image of a high-quality $\mathrm{PdCrO} 2$ thin film grown using 1ML-thick CuCrO2 buffer layer. Even though a 1ML-thick $\mathrm{CuCrO} 2$ buffer layer was used, there is no image contrast corresponding to the $\mathrm{Cu}$ atomic plane in the HAADF STEM image. The scale bar indicates $2 \mathrm{~nm}$.

References

1 P. J. Moll et al., Science 351, 1061-1064 (2016).

2 V. Sunko et al., Nature 549, 492-496 (2017).

3 H. Takatsu et al., Phys. Rev. Lett. 105, 137201 (2010).

4 J. M. Ok et al., Phys. Rev. Lett. 111, 176405 (2013).

5 J. M. Ok et al., APL Mater. 8, 051104 (2020). 